\title{
PEDESTRIAN-BUS ROUTE AND PICKUP LOCATION PLANNING FOR EMERGENCY EVACUATION
}

\author{
Weike $\mathrm{LU}^{1}$, Feng WANG ${ }^{2}$, Lan $\mathrm{LIU}^{3^{*}}$, Guojing $\mathrm{HU}^{4}$, Jiannan $\mathrm{MAO}^{5}$ \\ ${ }^{1,3,5}$ School of Transportation and Logistics, Southwest Jiaotong University, Chengdu, China \\ ${ }^{2}$ Ingram School of Engineering, Texas State University, San Marcos, United States \\ ${ }^{4}$ Dept of Mathematics and Statistical Sciences, Jackson State University, Jackson, United States
}

Submitted 25 November 2018; resubmitted 5 February 2019, 30 April 2019; accepted 24 May 2019; first published online 13 October 2020

\begin{abstract}
Planning for a bus-based regional evacuation is essential for emergency preparedness, especially for hurricane or flood prone urban environments with large numbers of transit-dependent or transit-captive populations. This paper develops an optimization-based decision-support model for pedestrian-bus evacuation planning under bus fleet, pedestrian and bus routes, and network constraints. Aiming to minimize the evacuation duration time, an optimization model is proposed to determine the optimal pickup nodes for evacuees to assemble using existing pedestrian routes, and to allocate available bus fleet via bus routes and urban road network to transport the assembled evacuees between the pickup nodes and designated public shelters. The numerical examples with two scenarios based on the Sioux Falls street network from North Dakota (United States) demonstrates that this model can be used to optimize the evacuation duration time, the location of pickup nodes and bus assignment simultaneously.
\end{abstract}

Keywords: emergency evacuation, pedestrian-bus network, pickup location optimization, bus resource constraint, safety shelters, evacuation duration time.

\section{Notations}

$b_{m, d}-$ a binary variable, which equals 1 if shelter $d$ is used to evacuate evacuees from pickup node $m$ by bus, and 0 otherwise;

$b c$ - maximum number of passengers in a bus;

$b n$ - the total number of buses;

$c b_{m, d}$ - the total time it takes $n_{m}$ buses to transport all assembled evacuees at pickup node $m$ to shelter $d$ by round trip mode;

$D$ - set of all shelters;

$d$ - shelter $d$, belonging to set of all shelters $D$;

$E$ - set of pedestrian links in a pedestrian network $G$;

$G$ - a pedestrian network $G(N, E)$, where $N$ is the set of nodes and $E$ is the set of links;

$i$ - node $i$, which belongs to the node set of a pedestrian network $G$;

$j$ - node $j$, which belongs to the node set of a pedestrian network $G$;

$(i, j)$ - index of the pedestrian link between adjacent node $i$ and $j,(i, j \in N)$; $k_{o, m}$ - a binary variable, which equals 1 if the evacuees at origin $o$ are evacuated to pickup node $m$, and 0 otherwise;

$M$ - set of possible pickup nodes;

$m$ - pickup node $m$, which belongs to set of possible pickup nodes $M$;

$N$ - the node set of a pedestrian network $G$;

$n_{m}$ - the assigned number of buses at pickup node $m$;

$O$ - set of all demand nodes;

$o$ - evacuation origin $o$, which belongs to set of all demand nodes $O$;

$p_{o}$ - evacuation demand generated from origin $o$;

$t_{i, j}$ - the pedestrian travel time of link $(i, j)$;

$t b_{m, d}$ - the shortest bus travel time from pickup node $m$ to shelter $d$;

$t c_{o}$ - the evacuation time when the evacuees at evacuation origin $o$ is evacuated to a shelter;

$t t_{m}$ - the number of bus trips needed for $n_{m}$ buses to transport all assembled evacuees of pickup node $m$ to a shelter;

${ }^{*}$ Corresponding author. E-mail: jianan_l@home.swjtu.edu.cn 
upper - the upper of all travel cost $t c_{o}$, is also the duration of the evacuation;

$x_{i, j, o, m}$ - a binary variable, which equals 1 if link $(i, j)$ is used to assign the pedestrian flow from evacuation origin $o$ to pickup node $m,(i, j) \in E$, and 0 otherwise;

$y_{m}$ - a binary variable, which equals 1 if node $m$ is chosen to serve as a pickup node, and 0 otherwise;

$Z$ - the optimization objective $z=$ upper.

\section{Introduction}

The efficient operation of public transit system during an emergency evacuation is of fundamental importance in the strategic planning and emergency management for an urban environment. In 2015, a major traffic accident occurred on Shanghai Metro Line 1, and 110 buses were used to evacuate the affected passengers and residents within three hours (CCTV 2017). In 2017, bus transit played vital roles in evacuating people at the subway stations when terrorist attacks occurred in Brussel and London (Henderson, Foster 2017; Hanna 2017). Compared with individual-based evacuation by driving private cars, bus-based evacuation has quite some distinct advantages and characteristics, especially in densely populated areas. Transit can not only move large numbers of evacuee units (humans, animals) from a disaster affected area with relatively few vehicle trips in a speedy and efficient manner but it can also induce as little traffic congestion as possible. Especially, some level of bus priorities (bus lane, special bus signal) in evacuation operation is of benefit to enhance the reliability of evacuation routes. The recent occurrences of emergency events due to extreme weathers and terrorist attacks threatening urban communities with high population densities have served as the motivation to study and develop a novel model of strategic planning of pedestrian-bus evacuation for an evacuation operation of an urban community when information regarding the threats is somewhat available in advance (Heydar et al. 2016).

This paper integrates pedestrian evacuation guidance and a bus pickup location problem, and presents a mathematical model to design evacuation routes from evacuation origins to safe areas. The mathematical model combines two networks: pedestrian network and bus network, where evacuees leave for pickup nodes from origins on feet and take buses to designated shelters from pickup nodes. In addition, bus resource constraint is considered in this paper. The objective is to minimize evacuation duration time, which is defined as the time it takes till the last person to be evacuated. In contrast to evacuation models for minimizing the total travel time of all evacuees, this model focuses on avoiding longer evacuation routes.

Although there are many research efforts devoted to emergency evacuation planning in the topic of either bus or pedestrian based evacuation, few studies combine pe- destrian route, pickup location and bus route together. Furthermore, this study attempts to optimize evacuation duration time under the constraint of bus quantity. Most closely related to this topic, the work of Heydar et al. (2016) and Zhang, Chang (2014) determined how the transit-dependent people should be assigned to pickup nodes and identified vehicle routes during the evacuation process, but they assumed that there are enough buses or vehicles to transit the assembled evacuees.

The remainder of this paper proceeds as follows: after the Introduction section, the relevant literatures and previous studies are reviewed in the "Background" section. In the "Modelling..." section, the model variables and modelling process are described. In "Experiments and results", a small sample network is used to show the effectiveness of the model and solution strategy. Finally, the paper concludes with a summary of observations and findings in "Conclusions".

\section{Background}

Though some significant contributions have been made to varied evacuation modelling in the past (Murray-Tuite, Wolshon 2013), there are only a limited number of studies discussing the use of public transit to evacuate people in emergency management (Liu, Yu 2012). The current studies approaching to this topic can be divided into two categories, the simulation-based approach and analytical approach. Using VISSIM (https://www.ptvgroup.com/en/solutions/ products/ptv-vissim) traffic simulation tool, Elmitiny et al. (2007) evaluated a current plan and alternative plans by the deployment of transit under an emergency situation in a transit facility such as a bus depot. To reach the best evacuation strategy, nine evacuation scenarios were simulated and analysed. Naghawi and Wolshon (2011) also analysed the performance of their bus evacuation strategies based on simulation software in different aspects.

Comparatively, the analytical approach has been widely used for generating evacuation plans under disaster circumstances (Murray-Tuite, Wolshon 2013). He et al. (2009) proposed a stochastic optimal modelling technique to generate an evacuation plan for transit-dependent residents in the event of natural disasters such as the hurricane. In the study of He et al. (2009), the transit evacuation operation was converted to a multi-stage location-routing problem with uncertain demands. For the uncertainty of evacuation demand, Kulshrestha et al. (2014) presented a decision-support model for transit-based evacuation planning, where the location and number of pickup nodes for evacuees to assemble is optimized by a mixed-integer program.

Another stream of researchers has developed some analytical approaches to obtain the best bus evacuation strategy. Chen and Chou (2009) proposed a bi-level optimization model to determine the waiting locations and corresponding shelters, where the model was extended to contraflow operations, waiting spots and service loca- 
tions. Bish (2011) summarized the Bus Evacuation Problem $(\mathrm{BEP})$ as a variant of the vehicle routing problem, and analysed the difference between two proposed mathematical programming models for this problem in detail. In particular, one of Bish (2011)'s models was to optimize the upper bound of evacuation routes, and its actual significance was to minimize the duration of an evacuation. Aiming at bus routes under BEP framework, Goerigk et al. (2015) and Dikas, Minis (2016) also improved the model and presented specific solution algorithms.

As discussed above, despite some important contributions of previous studies in BEP, the focus integrating evacuee guidance (from buildings or parking lots to pickup nodes) and bus route (from pickup nodes to shelters) became popular until recently. Heydar et al. (2016) presented a mathematical model to plan emergencies in a densely populated urban zone, and it is capable to assign evacuees from buildings or parking lots to pickup nodes and to route buses from pickup nodes to shelters. However, the optimization of evacuation duration time, the constraint of bus quantity and bus capacity was not considered in his study. To better highlight the characteristics of our evacuation model, we would like to further give a summary of several works that have strong correlations to our research.

In comparison to other related papers, our study has some characteristics shown in Table 1. First, in objective function, this paper pays more attention to minimizing evacuation duration time instead of seeking integration optimization, like minimizing the total travel time. Second, this paper integrates the pedestrian network and bus network, and furthermore, optimizes pedestrian and bus routes, pickup locations and shelters simultaneously. Third, bus quantity and capacity constraint are considered as well in this paper. When the total number of buses is insufficient to transport all evacuees once, the bus assignment and transport trips are optimized.

\section{Modelling: problem description and assumptions}

In this section, we describe the framework for pedestrian-bus evacuation planning and propose a mathematical programming formulation to minimize the evacuation duration time. The model determines the optimal pickup nodes for evacuees to assemble, and allocates available buses to transport the assembled evacuees from pickup locations to possible shelters.

The proposed integrated model is depicted in Figure 1 as a (complete) graph in which all evacuation origins, pickup nodes, and shelters are represented by shapes; roads are represented by lines in the network; roads are represented by lines in the network. All evacuees walk in pedestrian network and then are transited to safety shelters by bus in bus network. The intersection nodes of pedestrian network and bus network are pickup nodes, which are the walk destinations of evacuees in pedestrian network and also the bus origin nodes in bus network.

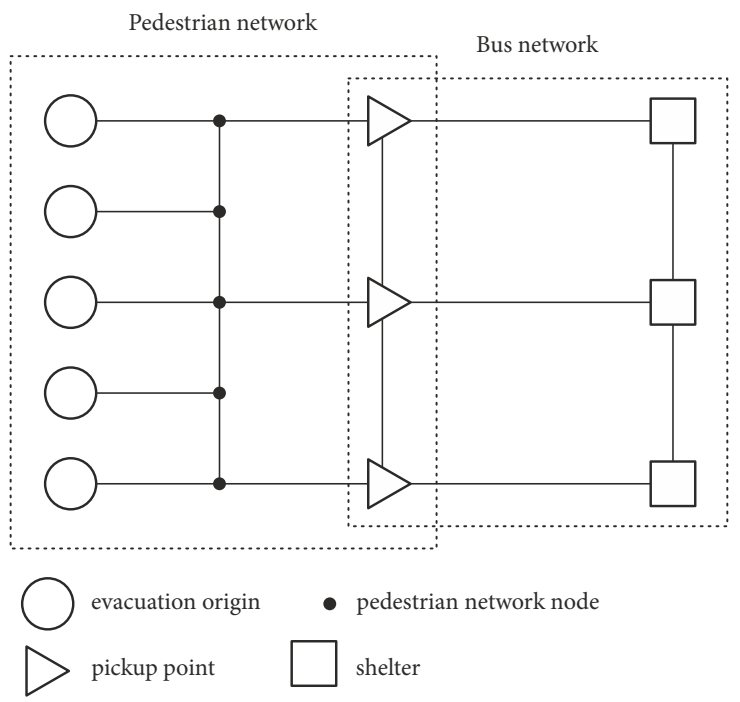

Figure 1. Sketch map of evacuation network

Table 1. Comparison of key elements in recently related evacuated model

\begin{tabular}{|l|l|c|l|}
\hline Objective function & \multicolumn{1}{|c|}{ Optimization type } & \multicolumn{1}{|c|}{ Pedestrian characteristic } & \multicolumn{1}{c|}{ Publication } \\
\hline MOBEBS & bus stop location & - & Kaisar et al. (2012) \\
\hline MTET & bus route & - & He et al. (2009) \\
\hline MTNE & vehicle assignment & pedestrian-vehicle mixed flows & Zhang, Chang (2014) \\
\hline MED & pickup location & - & Swamy et al. (2017) \\
\hline METD & evacuee assignment & uniform pedestrian flow and non-constraint & Liu, Yu (2012) \\
\hline MTNE & bus route & - & Li et al. $(2014)$ \\
\hline MET/MTET & bus route & - & Bish (2011) \\
\hline MTET & $\begin{array}{l}\text { pickup location; } \\
\text { pedestrian and bus route }\end{array}$ & uniform pedestrian flow and non-constraint & Heydar et al. (2016) \\
\hline MET & $\begin{array}{l}\text { pickup location; } \\
\text { pedestrian and bus route }\end{array}$ & $\begin{array}{l}\text { mixed pedestrian flow; } \\
\text { bus resource constraint }\end{array}$ & Current paper \\
\hline
\end{tabular}

MED - minimizing evacuation distance; MET - minimizing evacuation time; METD - minimizing the total distance; MOBEBS maximizing the overall benefit of evacuation bus stops; MTET - minimizing total evacuation time; MTNE - maximizing the total number of evacuees. 
Assumption (1) - at each pickup node, after all evacuees leaving for this node are assembled, then assigned buses begin to transport them. In view of this, how many the waiting evacuees can get on buses in each bus trip depends on the capacity of assigned buses whether they arrive early or late. Some pedestrian flows from different evacuation origin nodes are mixed at a selected pickup node, and therefore the bus use time/bus during time that the evacuee group of the different flows cost to their shelter is regarded to be equal.

Assumption (2) - the buses can arrive at the pickup node on time, and the travel time to the shelters is not affected by traffic congestion. For an effective evacuation, we think that the government arranges limited buses to participate in the event, and gives some priorities and traffic management strategies to keep bus lanes clear and unblocked. In addition, the shortest paths from any pickup nodes to any shelters are calculated in advance.

Assumption (3) - round trip mode. Based on limited bus transportation capacity, if the assembled evacuees on a pickup node are too many to transport to a shelter once by assigned buses, these buses can transport the evacuees by multiple trips. In addition, transfer time is not considered.

Assumption (4) - one-one-one evacuation. This paper proposes a model for the evacuation from one origin node to one pickup node, and to one shelter (one-oneone evacuation), which is similar to the work by Li et al. (2014). There are two reasons: (1) the evacuees are hard to be assigned to different routes quantitatively in realistic operation, so that we see the evacuees from an origin node as whole; (2) once a pickup node is determined, the bus from this node will leave for the closest shelter.

\section{Model formulation}

\subsection{Coupling constraints between pedestrian routes and pickup nodes}

To characterize the connection between pedestrian routes and pickup nodes, we introduce inequality (Equation (1)) to express the relationship between the location selection of a pickup node and potential pedestrian routes (Tong et al. 2015):

$$
k_{o, m} \leq y_{m}, \forall o \in O, m \in M .
$$

In addition, to guarantee at least one potential pickup node, inequality (Equation (2)) is used:

$$
\sum_{m \in M} y_{m} \geq 1 \text {. }
$$

\subsection{Pedestrian flow balance constraints}

In a pedestrian route, each pedestrian link flow corresponds to a single evacuation origin $o$, and pickup node $m$. To generate a route, a set of flow balance constraints are formulated as follows. It is worth noting that $k_{o, m}$ is a binary variable. If $k_{o, m}$ is equal to 0 , it means pickup node $m$ is not used, and there is no available pedestrian flow for pickup node $m$. If $k_{o, m}$ is equal to 1 , a route will be generated (Liu, Zhou, 2016).

$$
\begin{aligned}
& \sum_{j \in N} x_{i, j, o, m}-\sum_{j \in N} x_{j, i, o, m}= \\
& \begin{cases}k_{o, m}, & i=o,(i, j) \in E ; \\
-k_{o, m}, & i=m,(i, j) \in E ; \\
0, & i \neq o, i \neq m,(i, j) \in E,\end{cases} \\
& \forall o \in O, m \in M .
\end{aligned}
$$

\subsection{One-one-one evacuation constraints}

According to the Assumption 4, for any one of evacuation origins, only one pickup node is corresponding to it. In addition, for any one of pickup nodes, there is only one destination shelter. Equation (4) ensures the evacuation from one origin node to one pickup node in a pedestrian network. Equation (5) ensures the evacuation from one pickup node to one shelter in a bus network.

$$
\begin{aligned}
& \sum_{m \in M} k_{o, m}=1, \forall o \in O ; \\
& \sum_{d \in D} b_{m, d}=y_{m}, \forall m \in M .
\end{aligned}
$$

\subsection{Bus quantity constraints and bus using trips}

Equation (6) ensures that the total number of buses assigned to the pickup nodes is a fixed value. Inequality (Equation (7)) is used to ensure that all evacuees at pickup node $m$ are transported to a safety shelter, and the buses are fully loaded as possible, where $\sum_{o \in O} k_{o, m} \cdot p_{o}$ is the total number of evacuees at pickup node $m$. Equation (8) is used to calculate the time it takes to transport all evacuees from pickup node $m$ to shelter $d$ by $n_{m}$ buses with capacitated passengers under round trip mode.

$$
\begin{aligned}
& \sum_{m \in M} y_{m} \cdot n_{m}=b n \\
& t t_{m} \cdot n_{m} \cdot b c \geq \sum_{o \in O} k_{o, m} \cdot p_{o}, t t_{m} \cdot n_{m} \cdot b c \geq \sum_{o \in O} k_{o, m} \cdot p_{o} \\
& c b_{m, d}=\left(2 \cdot t t_{m}-1\right) \cdot t b_{m, d}, \forall m \in M, d \in D .
\end{aligned}
$$

\subsection{Upper bound of evacuation time for each evacuation origin and model objective function}

As shown in Equation (9), for evacuees at any one of the evacuation origin nodes, when they arrive at a shelter, the evacuation time is made up of two parts: travel time spent on pedestrian roads and bus using time. Constraint (Equation (10)) requires upper being greater or equalling to the maximum evacuation time of flows starting at evacuation origin nodes, which is then minimized by the objective function (Equation (11)). Apparently, constraint (Equation (9)) and constraint (Equation (10)) can be integrated as one constraint (Feng, Miller-Hooks 2014). 


$$
\begin{aligned}
& t c_{o}=\sum_{(i, j) \in E} \sum_{m \in M} x_{i, j, o, m} \cdot t_{i, j}+ \\
& \sum_{m \in M} \sum_{d \in D} k_{o, m} \cdot b_{m, d} \cdot c b_{m, d}, \forall o \in O ; \\
& t c_{o} \leq \text { upper, } \forall o \in O .
\end{aligned}
$$

The objective function is to minimize evacuation duration, given below:

$\min z=$ upper.

\section{Experiments and results}

According to constraint - Equations (7), (9) and (10), it is crystal clear that this model is a nonlinear programming problem with binary constraints. In this paper, the proposed optimization model was implemented in General Algebraic Modelling System (GAMS, $h t t p s: / / w w w$. gams.com) using Mixed Integer Non-Linear Programming (MINLP) solver. The computation experiments were conducted on a $2.2 \mathrm{GHz}$ YOGA computer with $8 \mathrm{~GB}$ of RAM. Sioux Falls street network from North Dakota (United States) has been used to test the effectiveness of the proposed method in some transportation science literatures (Lawphongpanich, Hearn 2004; Lownes et al. 2011). In view of the fact that this network is used for traffic network without pedestrians, we improve it as Figure 2 for different scenarios, and each link is marked with a freeflow travel cost and link attribute (pedestrian or bus using).

In this section: (1) Scenario 1 is used to test the existing optimal solution for this model and can be achieved by GAMS software; (2) Scenario 2 is used to demonstrate that the number of bus trips - bus assignment and multiple bus routes can be optimized simultaneously by this model.

\subsection{Scenario 1}

As shown in Figure 2a, there are three possible pickup nodes, five possible shelters and fifteen evacuation origin nodes with evacuation demand. Demand values at each demand node are shown in Table 2, and the bus information under Scenarios 1 and 2 is shown in Table 3.

The optimal result under Scenario 1 solved by GAMS software is shown in Table 4. Clearly, all 21 buses are assigned in the bus lane from pickup node 15 to shelter node 22 , therefore, evacuation time by bus is 9 time units. From Figure 3, it is easy to know that the evacuation route of origin 1 is the longest, and it costs 20 time units, which means the evacuation duration time of this pedestrianbus network is 20 time units. Exploring why node 15 in possible pickup node set is only chosen as an optimal pickup node, we suggest two contributing factors. Firstly, the minimal shortest route from possible pickup nodes to possible shelter nodes is the route from pickup node 15 to shelter node 22 . This means that the travel time of this route is much less than the others, and bus evacuation time will increase if the route is not chosen. Secondly, the total demand of all evacuation origin nodes is 630 evacuees, which can be just transported only once by 21 buses with 30 passenger capacity and it is hard to ensure one-time bus trip if 21 buses are separated and assigned to multiple pickup nodes to transport different-quantity evacuee groups. In addition, according to Figure 2a, we can find that all optimal pedestrian routes are the shortest paths from evacuation origin nodes to pickup nodes in the pedestrian network. In fact, it is not easy to manage 21 buses on one location in the same time. We do not consider bus delay for stopping and boarding, as this paper is in the context of simplifying static evacuation logistics.

b)

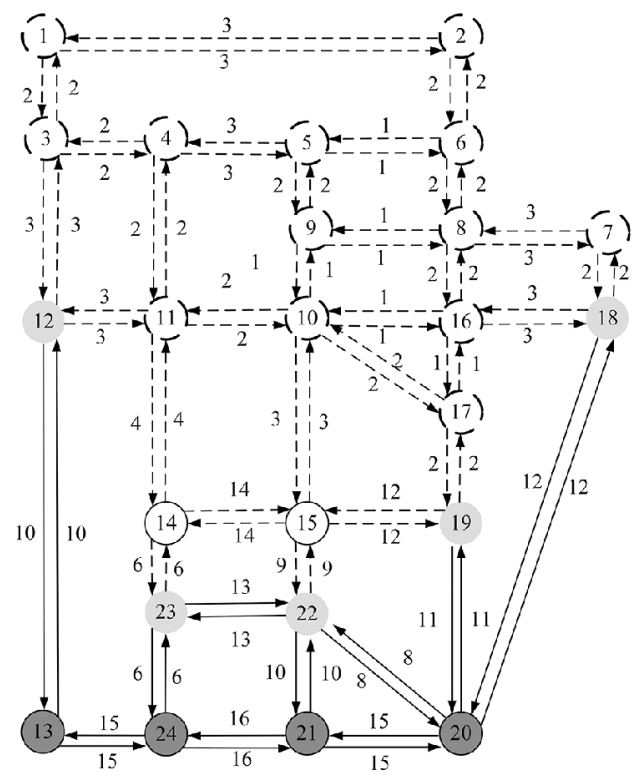

$\stackrel{9}{\longrightarrow}$ bus link and its travel time $\quad-\stackrel{1}{\rightarrow}$ pedestrian link and its travel time 
Table 2. Demand values at each demand node

\begin{tabular}{|l|c|c|c|c|c|c|c|c|c|c|c|c|c|c|c|c|}
\hline Demand nodes & 1 & 2 & 3 & 4 & 5 & 6 & 7 & 8 & 9 & 10 & 11 & 12 & 16 & 17 & 18 & Total demand \\
\hline Evacuees & 60 & 42 & 40 & 46 & 38 & 50 & 34 & 44 & 44 & 52 & 48 & 40 & 36 & 26 & 30 & 630 \\
\hline
\end{tabular}

Table 3. The bus information under Scenarios 1 and 2

\begin{tabular}{|l|l|}
\hline The number of available buses under Scenario 1 [veh] & 21 \\
\hline The number of available buses under Scenario 2 [veh] & 14 \\
\hline Bus capacity [pas/veh] & 30 \\
\hline
\end{tabular}

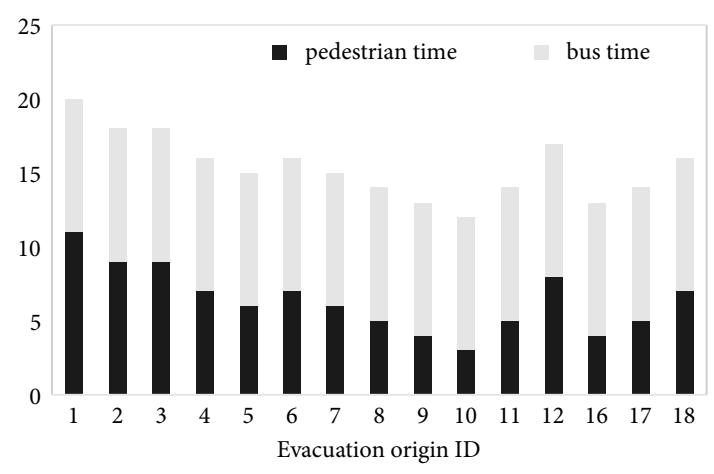

Figure 3. Evacuation during time for each evacuation origin under Scenario 1

However, cost time due to either bus scheduling or bus queuing can be easily added into the proposed model when necessary. For example, the bus travel time can integrate both actual travel time and average bus delay.

Also, in order to explain and clarify the result that the shortest evacuation duration time is 20 time units as GAMS software runs, the random experiments are designed by using MATLAB 2014 (https://www.mathworks. $\mathrm{com} /$ products/matlab.html), where $1000 \mathrm{random}$ evacuation schemes satisfying all constraint conditions are produced. In each evacuation scheme, evacuation origin nodes, pickup nodes to shelters, and selected shelters are randomly allocated. What's more, evacuation duration time is the time that the longest pedestrian-bus route costs. To satisfy constraint condition (Equation (3)) quickly and easily, Dijkstra's algorithm is used to calculate the route between any two nodes. The experiments results are illustrated in Figure 4a. It is not hard to see that the variation of evacuation duration time is volatile. When evacuation duration time ranks in descending order in Figure $4 \mathrm{~b}$, an obvious feature shows that all evacuation duration time is no less than 20 time units, which proves that the optimized result by GAMS is reasonable, and the proposed model in this paper can be used to minimize the upper bound of evacuation duration time. It is worth noting that there may be other evacuation routes except the routes in Table 4 when evacuation duration time is optimal. If their existence does not have an effect on optimal/maximal evacuation duration time, they will also be feasible routes. a)

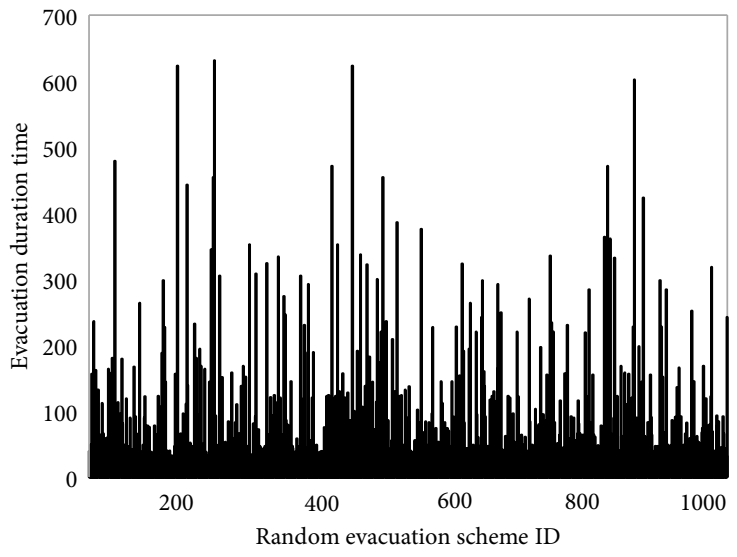

b)

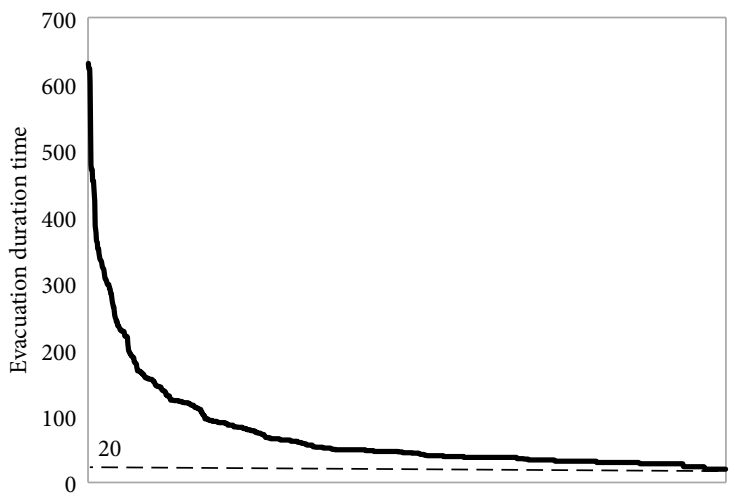

Figure 4. Results of random experiments: a - the evacuation duration time of random experiments; $b$ - ranked evacuation duration time in descending order

Table 4. Optimal results under Scenario 1

\begin{tabular}{|c|c|c|c|}
\hline $\begin{array}{c}\text { Evacuation } \\
\text { origin ID }\end{array}$ & $\begin{array}{l}\text { Pickup } \\
\text { location }\end{array}$ & $\begin{array}{l}\text { Pedestrian } \\
\text { route }\end{array}$ & $\begin{array}{l}\text { Bus } \\
\text { route }\end{array}$ \\
\hline 1 & \multirow{15}{*}{15} & $1-3-4-11-10-15$ & \multirow{15}{*}{$15-22$} \\
\hline 2 & & $2-6-5-9-10-15$ & \\
\hline 3 & & $3-4-11-10-15$ & \\
\hline 4 & & $4-11-10-15$ & \\
\hline 5 & & $5-9-10-15$ & \\
\hline 6 & & $6-5-9-10-15$ & \\
\hline 7 & & $7-8-9-10-15$ & \\
\hline 8 & & $8-9-10-15$ & \\
\hline 9 & & $9-10-15$ & \\
\hline 10 & & $10-15$ & \\
\hline 11 & & $11-10-15$ & \\
\hline 12 & & $12-11-10-15$ & \\
\hline 16 & & $16-10-15$ & \\
\hline 17 & & $17-16-10-15$ & \\
\hline 18 & & $18-16-10-15$ & \\
\hline
\end{tabular}




\subsection{Scenario 2}

In this scenario, the total number of buses is 14 . In order to make bus assignment more balanced, the bus travel time of the bidirectional links from node 12 to node 13 , from node 19 to node 20 , and from node 18 to node 20 are changed to 10,11 and 12 time units respectively. The set of possible pickup nodes are updated as node 12 , node 18 , node 19 , node 22 and node 23 , where node 12 and node 18 are not evacuation demand nodes anymore. The set of shelters are updated as node 13 , node 20 , node 21 and node 24 .

Because the passenger capacity of all 14 buses is less than the total evacuation demand, the optimization of bus transport trips has a more important effect on evacuation duration time. The results solved by GAMS are shown in Table 5 and Figure 5, and the running time is 10.23 s. Compared with Scenario 1, the number of evacuation routes and pickup nodes increase obviously in Scenario 2, which attributes to the travel time modification in some links and insufficient bus resource. As shown as Figure 5, the evacuation duration time is 34 time units, longer than that under Scenario 1. Especially, in Table 5, we can further find the evacuation routes going through nodes 9 , 10 and 11 are the most, which means that the evacuation efficiency will decrease deeply if the nodes are disturbed. These nodes should be paid more attention and implemented traffic management strategies for keeping node capacity and efficiency.

The results of bus assignment and transport trips under Scenario 2 are shown in Figure 6, and the bus assignment at pickup node 19 is maximum. Analysing this phenomenon, the reason is that the pickup nodes, linked by those longer pedestrian routes, may be allocated to more buses to reduce bus transport trips. In addition, the pickup nodes linked by the shorter bus lane to the shelters may be allocated to fewer buses to increase bus transport trips, saving the number of buses for other pickup nodes and this may reduce the integral evacuation duration time throughout the pedestrian-bus network.

Table 5. Optimal results under Scenario 2

\begin{tabular}{|c|c|l|c|}
\hline $\begin{array}{c}\text { Evacuation } \\
\text { origin ID }\end{array}$ & $\begin{array}{c}\text { Pickup } \\
\text { location }\end{array}$ & \multicolumn{1}{|c|}{$\begin{array}{c}\text { Pedestrian } \\
\text { route }\end{array}$} & $\begin{array}{c}\text { Bus } \\
\text { route }\end{array}$ \\
\hline 1 & 12 & $1-3-12$ & $12-13$ \\
\hline 3 & 12 & $3-12$ & $12-13$ \\
\hline 16 & 12 & $16-10-11-12$ & $12-13$ \\
\hline 17 & 12 & $17-10-11-12$ & $12-13$ \\
\hline 2 & 18 & $2-6-8-16-18$ & $18-20$ \\
\hline 7 & 18 & $7-18$ & $18-20$ \\
\hline 9 & 18 & $9-10-16-18$ & $18-20$ \\
\hline 4 & 19 & $4-11-10-17-19$ & $19-20$ \\
\hline 5 & 19 & $5-9-10-17-19$ & $19-20$ \\
\hline 6 & 19 & $6-8-16-17-19$ & $19-20$ \\
\hline 10 & 22 & $10-15-22$ & $22-20$ \\
\hline 8 & 23 & $8-9-10-11-14-23$ & $23-24$ \\
\hline 11 & 23 & $11-14-23$ & $23-24$ \\
\hline
\end{tabular}

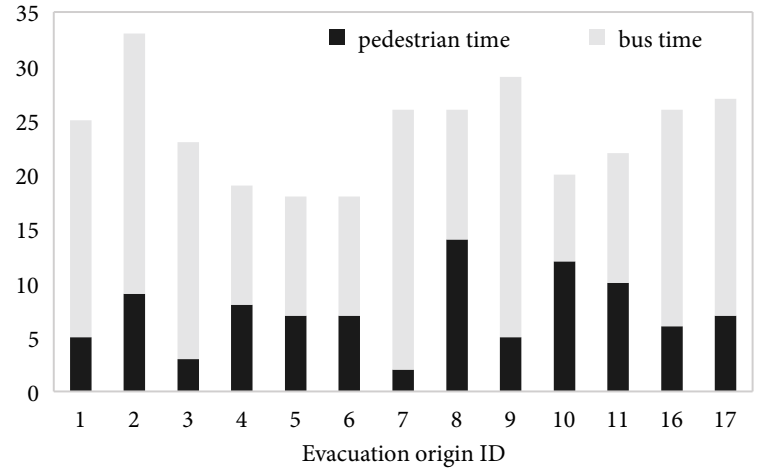

Figure 5. Evacuation during time for each evacuation origin under Scenario 2

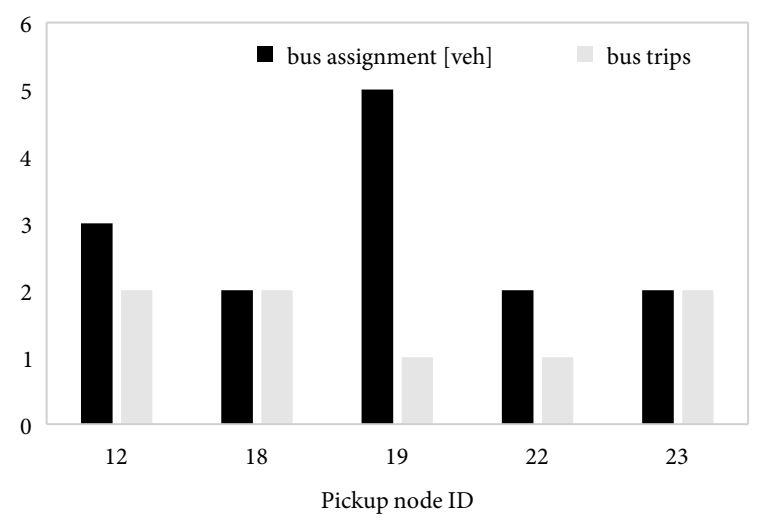

Figure 6. Results of bus assignment and transport trips under Scenario 2

\section{Conclusions}

The paper differs from its closest relatives in two important aspects: (1) the objective function, which is to minimize the duration of an evacuation, that is, how long it takes to transport evacuees from origins to shelters in the pedestrian-bus network; (2) bus transport trips, bus assignments and bus routes can be optimized simultaneously in this model. Aiming to minimize the evacuation duration time, this paper presents an approach to determine the location of pickup nodes for evacuees to assemble in a pedestrian-bus network. Moreover, this method is considered to be applied under a long/short-notice evacuation, especially when the bus resource is constrained. Through experimental tests in the Sioux Falls street network from North Dakota (United States), the proposed model is verified as reasonable for optimizing the assignment of bus resources to evacuate pedestrians. GAMS software is used to solve the model under two designed scenarios. The results show that: (1) in the condition of current network size, the high-quality solution for the proposed model can be attained by GAMS; (2) when the bus capacity is limited, the optimized bus schedule tends to allocate more buses to pickup nodes with larger demand and longer evacuation routes, and assign less buses with more transit round trips to pickup nodes with shorter evacuation routes.

Although we have provided mathematical programming formulations for the pedestrian-bus route and pickup location optimization with bus resource constraints 
under evacuation circumstances, due to its computational complexity, a heuristic technique is required for realistic size problems, which will be our next research goal. There are still two challenges for the study in the future. First, to provide an effective and accurate evacuation plan, the dynamic delay in pedestrian-bus transfer process should be considered. For this challenge, it may be a suitable solution to abstract important activities (such as pickup, walk, drop off) as pedestrian-state-time vertexes to construct pedestrian-state-time networks and then to solve the multidimensional problem. Second, the time dependent effect is not considered in this model. If the time dependent effect is included, a dynamic bus dispatch plan would be proposed.

\section{Acknowledgements}

This work is supported by National Natural Science Foundation of China (General Program, Grant No 61873216).

\section{Funding}

This work was supported by the National Natural Science Foundation of China (General Program, Grant No 61873216).

\section{Author contributions}

The authors confirm contribution to the paper as follows: "'m study conception and design: Weike $\mathrm{Lu}$;

"») data collection: Feng Wang;

"» analysis and interpretation of results: Weike Lu, Lan Liu;

»» draft manuscript preparation: Guojing Hu, Jianan Mao.

All authors reviewed the results and approved the final version of the manuscript.

\section{Disclosure statement}

There are no conflicts of interest.

\section{References}

Bish, D. R. 2011. Planning for a bus-based evacuation, OR spectrum 33(3): 629-654. https://doi.org/10.1007/s00291-011-0256-1

CCTV. 2017. A power supply failure happens in Shang metro, CCTV News 14 April 2017. Available from Internet: http://news.cctv.com/2017/04/14/ARTI3Bj1nhIeJ6chNAUwaSO1170414.shtml (in Chinese).

Chen, C.-C.; Chou, C.-S. 2009. Modeling and performance assessment of a transit-based evacuation plan within a contraflow simulation environment, Transportation Research Record: Journal of the Transportation Research Board 2091: 40-50. https://doi.org/10.3141/2091-05

Dikas, G.; Minis, I. 2016. Solving the bus evacuation problem and its variants, Computers \& Operations Research 70: 75-86. https://doi.org/10.1016/j.cor.2015.12.012
Elmitiny, N.; Ramasamy, S.; Radwan, E. 2007. Emergency evacuation planning and preparedness of transit facilities: traffic simulation modeling, Transportation Research Record: Journal of the Transportation Research Board 1992: 121-126. https://doi.org/10.3141/1992-14

Feng, L.; Miller-Hooks, E. 2014. A network optimization-based approach for crowd management in large public gatherings, Transportation Research Part C: Emerging Technologies 42: 182-199. https://doi.org/10.1016/j.trc.2014.01.017

Goerigk, M.; Deghdak, K.; T'Kindt, V. 2015. A two-stage robustness approach to evacuation planning with buses, Transportation Research Part B: Methodological 78: 66-82. https://doi.org/10.1016/j.trb.2015.04.008

Hanna, J. 2017. The London train explosion is the latest of 5 terror incidents in 2017 in the UK, CNN News 15 September 2017. Available from Internet: https://edition.cnn. com/2017/09/15/world/uk-terror-events-2017/index.html

He, S.; Zhang, L.; Song, R.; Wen, Y.; Wu, D. 2009. Optimal transit routing problem for emergency evacuations, in TRB 88th Annual Meeting Compendium of Papers DVD, 11-15 January 2009, Washington, DC, US. 13 p.

Henderson, B.; Foster, P. 2017. Brussels station blast: suspect 'wearing explosive belt' shot dead by troops, The Telegraph 21 June 2017. Available from Internet: https://www.telegraph. co.uk/news/2017/06/20/traffic-halted-brussels-small-explosion-central-train-station/

Heydar, M.; Yu, J.; Liu, Y.; Petering, M. E. H. 2016. Strategic evacuation planning with pedestrian guidance and bus routing: a mixed integer programming model and heuristic solution, Journal of Advanced Transportation 50(7): 1314-1335. https://doi.org/10.1002/atr.1403

Kaisar, E. I.; Hess, L.; Portal Palomo, A. B. 2012. An emergency evacuation planning model for special needs populations using public transit systems, Journal of Public Transportation 15(2): 45-69. http://doi.org/10.5038/2375-0901.15.2.3

Kulshrestha, A.; Lou, Y.; Yin, Y. 2014. Pick-up locations and bus allocation for transit-based evacuation planning with demand uncertainty, Journal of Advanced Transportation 48(7): 721733. https://doi.org/10.1002/atr.1221

Lawphongpanich, S.; Hearn, D. W. 2004. An MPEC approach to second-best toll pricing, Mathematical Programming 101(1): 33-55. https://doi.org/10.1007/s10107-004-0536-5

Li, G.; Zhang, L.; Wang, Z. 2014. Optimization and planning of emergency evacuation routes considering traffic control, The Scientific World Journal 2014: 164031. http://doi.org/10.1155/2014/164031

Liu, J.; Zhou, X. 2016. Capacitated transit service network design with boundedly rational agents, Transportation Research Part B: Methodological 93: 225-250. https://doi.org/10.1016/j.trb.2016.07.015

Liu, Y.; Yu, J. 2012. Emergency evacuation planning for highly populated urban zones: a transit-based solution and optimal operational strategies, in B. Eksioglu (Ed.). Emergency Management, 59-74. https://doi.org/10.5772/33653

Lownes, N. E.; Wang, Q.; Ibrahim, S.; Ammar, R. A.; Rajasekaran, S.; Sharma, D. 2011. Many-to-many game-theoretic approach for the measurement of transportation network vulnerability, Transportation Research Record: Journal of the Transportation Research Board 2263: 1-8.

https://doi.org/10.3141/2263-01

Murray-Tuite, P.; Wolshon, B. 2013. Evacuation transportation modeling: an overview of research, development, and practice, Transportation Research Part C: Emerging Technologies 27: 25-45. https://doi.org/10.1016/j.trc.2012.11.005 
Naghawi, H.; Wolshon, B. 2011. Performance of multi-modal evacuation traffic networks: a simulation based assessment, in TRB 90th Annual Meeting Compendium of Papers DVD, 23-27 January 2011, Washington, DC, US. 21 p.

Swamy, R.; Kang, J. E.; Batta, R.; Chung, Y. 2017. Hurricane evacuation planning using public transportation, Socio-Economic Planning Sciences 59: 43-55.

https://doi.org/10.1016/j.seps.2016.10.009

Tong, L.; Zhou, X.; Miller, H. J. 2015. Transportation network design for maximizing space-time accessibility, Transportation Research Part B: Methodological 81(2): 555-576. https://doi.org/10.1016/j.trb.2015.08.002

Zhang, X.; Chang, G. L. 2014. An optimization model for guiding pedestrian-vehicle mixed flows during an emergency evacuation, Journal of Intelligent Transportation Systems: Technology, Planning, and Operations 18(3): 273-285.

https://doi.org/10.1080/15472450.2013.824763 